\title{
Revisión bibliográfica en idioma español sobre el arteterapia como herramienta psicoterapéutica ${ }^{4}$
}

\author{
Alejandro Barbosa González \\ Magister en Psicología Clínica y de la familia. \\ Universidad Antonio Nariño, Colombia \\ Correo electrónico: abarbosagonzalez@gmail.com
}

Sofía Alexandra Garzón Novoa

Psicóloga.

Universidad Antonio Nariño, Colombia

Correo electrónico: sofyygarzon@gmail.com
Recibido: 20/01/2019

Evaluado: $20 / 03 / 2019$

Aceptado: 27/03/2019

\section{Resumen}

El objetivo del estudio consistió en realizar una revisión bibliográfica en lengua española sobre el arteterapia como herramienta psicoterapéutica desde el año 2006 a 2017. Las bases de datos consultadas fueron Dialnet, Ebsco Host, El Psitio, Elsevier, Google Académico, Google Books, Red Visual, Redalyc, ResearchGate, Salud Mental y Scielo. Los descriptores: "Arteterapia", "terapia" "artística", "terapia de arte", "psicología" y "arte"; "psicoterapia" y "arte"; "creatividad" y "psicología"; "creatividad" y "terapia". En total se encontraron 105 documentos, de los que se pre-seleccionaron 73 $y$, finalmente, se seleccionaron 44. Los hallazgos evidencian que el $66 \%$ de documentos corresponden a estudios empíricos y el $34 \%$ a revisiones documentales. También aparecen 26 problemáticas, siendo los trastornos del neurodesarrollo los más trabajados con la terapia artística. En segundo lugar, se encuentran los trastornos psicóticos. Se concluye que las técnicas artísticas más utilizadas en las diferentes problemáticas que aparecen en los documentos seleccionados fueron el dibujo, pintura y modelado con arcilla. Igualmente, que el arteterapia más que ser una terapia propia de la psicología, ha buscado ser una terapia interdisciplinar, por lo que muchos profesionales especializados provienen de carreras como Bellas artes, Terapia cupacional, Trabajo social, Psiquiatría y Enfermería, entre otras.

Arteterapia, creatividad, psicología, arte, revisión bibliográfica

4 Para citar este artículo: Barbosa, A., \& Garzón, S.A. (2020). Revisión bibliográfica en idioma español sobre el arteterapia como herramienta psicoterapéutica. Informes Psicológicos, 20(2), pp. 55-65 http://dx.doi.org/10.18566/ infpsic.v20n2a4 


\section{Literature review in Spanish language about art therapy as a psychotherapeutic tool}

\section{Abstract}

The objective of the study was to carry out a literature review in Spanish about art therapy as a psychotherapeutic tool. The study took place from 2006 to 2017. The databases consulted were Dialnet, EBSCOhost, El Psitio, Elsevier, Google Scholar, Google Books, Red Visual, Redalyc, ResearchGate, Salud Mental and Scielo; and the descriptors reviewed were "Art therapy," "artistic" "therapy," "therapy of art," "psychology," and "art"; "psychotherapy" and "art"; "creativity" and "psychology"; "creativity" and "therapy". In total, 105 documents were found, of which 73 were pre-selected and, finally, 44 were selected. The findings show that $66 \%$ of documents correspond to empirical studies and $34 \%$ to documentary reviews. There are also 26 problems. Neurodevelopmental disorders are the most worked using artistic therapy. Additionally, there are psychotic disorders. It is concluded that the most used artistic techniques in the different problems that appear in the selected documents were drawing, painting and modeling with clay. Likewise, it was found that art therapy has sought to be an interdisciplinary therapy rather than a therapy of psychology, which is why many specialized professionals come from careers such as Fine Arts, Occupational Therapy, Social Work, Psychiatry and Nursing, among others.

Keywords

\section{Revisão bibliográfica em espanhol da arteterapia como ferramenta psicoterapêutica}

Resumo

0 objetivo do estudo consistiu em fazer uma revisão bibliográfica em espanhol ao respeito da arteterapia como ferramenta psicoterapêutica a partir do ano 2006 até 2017. As bases de dados consultadas foram Dialnet, Ebsco Host, El Psitio, Elsevier, Google Scholar, Google Livros, Red Visual, Redalyc, ResearchGate, Salud Mental e Scielo. Como descritores: "Arteterapia", "terapia" "artística", "terapia de arte", "psicología" y "arte"; "psicoterapia" y "arte"; "creatividad" y "psicología"; "creatividad" y "terapia". Achou-se um total de 105 documentos, entre os quais se fez uma pré-seleção de 73, e, finalmente escolheram-se 44 . 0 s resultados mostram que $66 \%$ dos documentos correspondem a estudos empíricos e $34 \%$ a revisões documentais. Existem também 26 problemas, sendo as desordens do desenvolvimento neurológico os mais trabalhados com a terapia artística, seguidos pelos distúrbios psicóticos. Conclui-se que as técnicas artísticas mais utilizadas nos diferentes problemas que aparecem nos documentos selecionados foram desenho, pintura e modelagem com argila. Evidencia-se também que a arteterapia tem procurado se perfilar como uma terapia interdisciplinar e não somente psicológica, razão pela qual muitos profissionais especializados vêm de carreiras como Belas Artes, Terapia Ocupacional, Serviço Social, Psiquiatria e Enfermagem, entre outros. 


\section{ntroducción}

Para hablar de arteterapia es importante definir por separado arte y psicoterapia, ya que esto contextualiza al lector y le permite integrar la comprensión de la temática. En cuanto al arte, es definido por Coll (2015) como el "resultado de la unión entre el autor y su producto, es decir, su obra..., el arte es, esencialmente, un modo de comunicación que puede variar dependiendo del tipo de arte y de obra que se haga" (p. 221). También Sánchez (2003) indica que es la forma estética de expresión de los sentimientos, trasmitiéndose desde un acto visual como auditivo. Por el lado de la psicoterapia, Strupp (1978) la concibe como un proceso interpersonal destinado a promover el cambio de sentimientos, cogniciones, actitudes y conductas; las cuales resultan problemáticas para el individuo que busca ayuda de un profesional entrenado. También Benito (2009) considera a la psicoterapia como un procedimiento, el cual debe "basar sus intervenciones en una teoría científica del funcionamiento y cambio conductual/psicológico de las personas y presentar un conjunto organizado de técnicas, capacidades o formas de promover una mejora en las condiciones que generan el motivo de consulta" (p. 2).

En definitiva, la utilización del arte como estrategia de intervención dentro de los procesos psicoterapéuticos, es una forma de flexibilizar y adaptar dichos procesos a las posibilidades expresivas de las personas. Deliberada o intencionalmente, las personas buscan formas creativas para expresar sus emociones. Lo interesante surge en el momento en que sistematizamos este tipo de expresiones y las llevamos a prueba para validarlas y formalizarlas. Tanto la psicoterapia como el arte se valen de la creatividad en diferentes momentos. De la misma manera, las emociones se encuentran presentes en ambos casos. El arte y la intervención psicoterapéutica han logrado convergencias interesantes que se relacionan con el bienestar de las personas y la salud.

\section{Apuntes históricos sobre arteterapia}

Si bien el ser humano siempre ha hecho uso del arte para fines terapéuticos, autores como Hogan (2001) consideran que sus inicios se remontan a los años 40 del siglo XX, después de la Segunda Guerra Mundial. En palabras del autor: "La terapia empleando el trabajo de creación de imágenes se llevó a cabo antes de esa fecha en el contexto del tratamiento moral, el psicoanálisis y posiblemente como parte de experimentos en el arte moderno" (p. 25). El nacimiento del arteterapia se presenta de forma simultánea en Inglaterra con Adrián Hill y en Estados Unidos con Margaret Naumburg. En cuanto al origen británico, Hill (1945) acuña por vez primera el término de Arteterapia, tras estar ingresado en 1938 en King Edward VII Sanatorium por causa de una tuberculosis. Por medio de procesos creativos, Hill exploró sus vivencias y encontró una forma para su recuperación emocional. También apoyó a otros pacientes (en especial soldados) para que expresaran asuntos relacionados con la ansiedad causada por la enfermedad y sufrimiento experimentado durante la guerra (Coll, 2015). Para el caso estadounidense, se 
considera a Margaret Naumburg y Edith Kramer, las principales precursoras en el desarrollo y teorización sobre el tema. Junge y Asawa (1994) recuerdan que el arteterapia proviene de una tradición psicoanalítica, donde la expansión del mismo suscitó que artistas y personas del campo educativo como Naumburg y Kramer se psicoanalizaran y combinaran la experiencia con habilidades propias de la pedagogía y el arte. También López (2009) resalta que Naumburg no solo se enfocó en teorizar sobre arteterapia y exponer el uso del arte como terapia, sino que trabajó para sacar del anonimato esta disciplina por medio de la presentación de varias de sus obras en diversos congresos de psiquiatría. Por su parte, Kramer se interesó por realizar la distinción de la independencia entre el uso del arte en la psicoterapia y arteterapia.

\section{Beneficios del arteterapia y creatividad}

La terapia artística o arteterapia es distinguida cada vez más como método que da paso a la comunicación no verbal, solución de conflictos emocionales, fomento del desarrollo personal, autoconciencia y medio complementario para el tratamiento de trastornos mentales (Vásconez, 2017). Según Fabres (2011), los beneficios del arteterapia como tratamiento de personas que presentan algún tipo de psicosis, se ven reflejados en un desarrollo de la concentración, destreza manual y creativa. Así mismo, en una mayor autonomía dentro de un contexto controlado, otorgando la capacidad para contemplar y analizar lo plasmado. También Dumas y Aranguren (2013) acotan que:
El arteterapia resulta un dispositivo que promueve el crecimiento personal en todas sus dimensiones: Emocional, cognitivo, social y físico... Puede ser aplicado en diferentes contextos siendo un recurso flexible capaz de ser utilizado a nivel grupal como individual, en contextos escolares, hospitalarios, o en la clínica privada (p. 44).

Para estos mismos autores, el arteterapia permite asumir actitudes que contribuyen con un sentido de vida, lo cual reduce los síntomas de depresión y favorece una flexibilidad cognitiva que enriquece los canales de comunicación. Finalmente, García (2018) resume una lista de beneficios de manera general. Allí aparece la liberación por medio de la expresión de sentimientos, reforzamiento de la autoimagen/ autoestima y mayor efectividad al tratar trastornos alimentarios e imagen corporal, entre otros. En general, los beneficios no solamente se pueden observar en casos de desórdenes mentales o del desarrollo, sino también en cambios pequeños en la calidad de vida de las personas. Todo lo anterior no sería posible sin la participación de los procesos creativos dentro de las intervenciones.

En cuanto a la creatividad, Aristóteles la consideraba como un proceso racional. Dicho proceso tendría sus orígenes en leyes naturales, resaltando la predisposición de los grandes poetas y otros artistas a la melancolía (Aurora \& Lara, 2000). Desde una óptica psicológica, se constituye en referente para la evaluación e intervención en diferentes ámbitos como el clínico, organizacional y educativo, entre otros (Corbalán, 2006). Uno de los autores que da paso a la creatividad dentro de la psicología fue Guilford, quien motivaba para que se 
investigara más y se evaluara, proponiendo que se podría medir de la misma forma como se hacía con la inteligencia; llegando a establecer una relación importante entre estos dos conceptos (Obradors, 2007). Otra definición es la que propone Vecina (2006): "Crear es inventar posibilidades, es un ejercicio de libertad que en el reino animal solo el cerebro humano puede desarrollar porque no está determinado por los estímulos externos, sino por los proyectos y metas que el mismo crea" (p. 32).

La creatividad y el arteterapia son dos conceptos bastante cohesionados, ya que el arteterapia se vale de la creatividad del consultante y el terapeuta. Allí se produce una construcción conjunta que orienta los procesos de intervención y cambio. Dichos procesos contemplan la implementación de estrategias orientadas por técnicas artísticas, las cuales dan cuenta de la relación establecida entre el terapeuta y la persona que lo consulta.

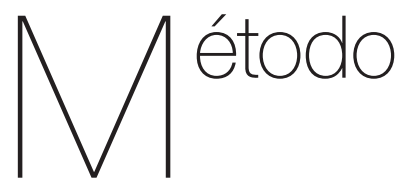

El estudio es de carácter documental. Existen diferentes tipos de estudios documentales como las revisiones sistemáticas, bibliométricas y bilbiográficas ente otras. Para el caso del presente trabajo la revisión implementada es bibliográfica. Dentro de las ventajas de la revisión bibliográfica se encuentra la recopilación y depuración de la información para su análisis. De igual forma, la categorización y organización para flexibilizar su consulta a otros investigadores o personas interesadas en la temática abordada. Finalmente, se pueden realizar hipótesis o plantear preguntas de investigación encaminadas a la replicación de estudios (García, 2007; Vanegas, Barbosa \& Pedraza 2017).

Con respecto a los objetivos y hablando en términos generales, se pretendía realizar una revisión bibliográfica de documentos en lengua española sobre el arteterapia como herramienta psicoterapéutica desde el año 2006 a 2017. A nivel más específico, analizar la eficacia de las técnicas y enfoque de formación de los profesionales que utilizan el arteterapia. También determinar en qué problemáticas se ha aplicado y describir los tipos de documentos encontrados. Finalmente, identificar el país de publicación y la procedencia de los autores.

\section{Procedimiento}

Para la búsqueda de los documentos se definieron unos criterios de inclusión (documentos en español, en un rango de tiempo de 2006 a 2017, corresponder a libros, revistas científicas, trabajos de pregrado, maestría y doctorado). En primer lugar, se consultó la Red Distrital de Bibliotecas Públicas de Bogotá. Después se realizó una búsqueda en diferentes bases de datos y bibliotecas virtuales pertenecientes a universidades de Colombia como la Pontificia Universidad Javeriana, Universidad Antonio Nariño y Universidad Nacional de Colombia. Posteriormente, se identificaron repositorios universitarios internacionales como el de la Universidad Abierta Iberoamericana, Universidad Complutense de Madrid, Universidad de Murcia, Universidad de Valladolid y Universitat Politècnica de València. También 
se ubicaron documentos en las bases de datos (Dialnet, Ebsco Host, El Psitio, Elsevier, Google Académico, Google Books, Red Visual, Redalyc, ResearchGate, Salud Mental y Scielo). Para la búsqueda se utilizaron los siguientes descriptores: "Arteterapia", "terapia artística", "terapia de arte", "psicoterapia y arte"; "creatividad y psicología"; "creatividad y terapia". Con los documentos encontrados se realizó una preselección y selección, gracias a un proceso de categorización inductiva.

\section{Análisis de resultados}

Se encontraron 105 documentos, se pre-seleccionaron 73 y finalmente se seleccionaron 44. Para el análisis de la información se construyeron diferentes matrices. El análisis contempló el tipo de documento, la formación de los profesionales, problemáticas trabajadas, país de publicación, procedencia de los autores y eficacia de las técnicas utilizadas. A continuación, se presentan algunas tablas con el fin de visualizar y ampliar mejor los resultados. Allí se destacan aspectos como la escasa publicación en revistas de Suramérica y la dominancia de revistas y repositorios españoles. En Sudamérica aparecen Chile y Colombia como los países donde más se han publicado documentos sobre el tema. En cuanto a las problemáticas abordadas, se encuentra, en primer lugar, el tema del neurodesarrollo y, en segundo, el espectro de la esquizofrenia y otros trastornos psicóticos. Finalmente, los trastornos depresivos.

Tabla 1

Cantidad de documentos según revistas

\begin{tabular}{|c|c|c|c|c|}
\hline Revista & Pre-seleccionados & Seleccionados & Empíricos & Documental \\
\hline Arte, Individuo y Sociedad & 5 & 3 & 2 & 1 \\
\hline Internacional Journal of Developmental and Educational Psychology & 1 & 1 & 1 & 0 \\
\hline $\begin{array}{l}\text { Investigaciones Feministas: Papales de Estudios } \\
\text { de Mujeres, Feministas y de Género }\end{array}$ & 1 & 1 & 1 & 0 \\
\hline $\begin{array}{l}\text { Arteterapia. Papeles de Arteterapia y Educación } \\
\text { Artística Para la Inclusión Social }\end{array}$ & 16 & 13 & 11 & 2 \\
\hline $\begin{array}{l}\text { Informaciones Psiquiátricas: Publicación Científica } \\
\text { de los Centros de la Congregación de Hermanas } \\
\text { Hospitalarias del Sagrado Corazón de Jesús }\end{array}$ & 1 & 1 & 0 & 1 \\
\hline $\begin{array}{l}\text { Educación y Futuro: Revista de Investigación } \\
\text { Aplicada y Experiencias Educativas }\end{array}$ & 1 & 0 & 0 & 0 \\
\hline $\begin{array}{l}\text { Publicaciones Didácticas Revista Profesional de } \\
\text { Investigación, Docencia y Recursos Didácticos }\end{array}$ & 1 & 1 & 0 & 1 \\
\hline Psicooncología & 1 & 1 & 0 & 1 \\
\hline Sophia Educación & 3 & 1 & 0 & 1 \\
\hline Papeles de Trabajo Sobre Cultura, Educación y Desarrollo Humano & 1 & 1 & 1 & 0 \\
\hline Centros de Estudios en Diseño y Comunicación & 1 & 1 & 0 & 1 \\
\hline Artseduca & 1 & 0 & 0 & 0 \\
\hline
\end{tabular}




\begin{tabular}{|c|c|c|c|c|}
\hline Revista & Pre-seleccionados & Seleccionados & Empíricos & Documental \\
\hline $\begin{array}{l}\text { CESAG Centro de Enseñanza Superior Alberta Giménez } \\
\text { Adscrito a la Universidad Pontificia Comillas }\end{array}$ & 1 & 0 & 0 & 0 \\
\hline Enfermería Global & 1 & 1 & 1 & 0 \\
\hline Reflexiones & 1 & 0 & 0 & 0 \\
\hline Terapia Psicológica & 1 & 0 & 0 & 0 \\
\hline Revista Atticus & 1 & 0 & 0 & 0 \\
\hline Pensamiento e Investigación Social & 1 & 0 & 0 & 0 \\
\hline Psicoperspectivas. Individuo y Sociedad & 1 & 0 & 0 & 0 \\
\hline Salud Mental & 1 & 0 & 0 & 0 \\
\hline Byzantion Nea Hellás & 1 & 0 & 0 & 0 \\
\hline Revista Electrónica Interuniversitaria de Formación del Profesorado & 1 & 1 & 0 & 1 \\
\hline Red Visual & 1 & 0 & 0 & 0 \\
\hline Psicología 02549247 & 1 & 1 & 1 & 0 \\
\hline Gestalt Boletín de la AETG & 1 & 0 & 0 & 0 \\
\hline La Biblioteca Cohrane Plus & 1 & 1 & 0 & 1 \\
\hline \multirow[t]{2}{*}{ Total } & 47 & 28 & 18 & 10 \\
\hline & & & Total & 28 \\
\hline
\end{tabular}

Tabla 2

Repositorios universitarios

\begin{tabular}{|c|c|c|c|c|}
\hline Repositorios universitarios & Pre-seleccionados & Seleccionados & Empíricos & Documental \\
\hline Pontificia Universidad Javeriana & 2 & 1 & 0 & 1 \\
\hline Universidad Central del Ecuador & 1 & 0 & 0 & 0 \\
\hline Universidad de Murcia & 2 & 1 & 1 & 0 \\
\hline Universidad de Salamanca & 1 & 0 & 0 & 0 \\
\hline Universidad de Chile & 3 & 3 & 3 & 0 \\
\hline Universidad Complutense de Madrid & 2 & 1 & 1 & 0 \\
\hline Universitat de Lleida & 1 & 0 & 0 & 0 \\
\hline Universidad de Valladolid & 2 & 2 & 2 & 0 \\
\hline Universitat Politècnica de València & 2 & 1 & 1 & 0 \\
\hline Universidad Abierta Iberoamericana & 1 & 0 & 0 & 0 \\
\hline \multirow[t]{2}{*}{ Total } & 17 & 9 & 8 & 1 \\
\hline & & & Total & 9 \\
\hline
\end{tabular}


Tabla 3

Capítulos de libro

\begin{tabular}{lcccc}
\hline \multicolumn{1}{c}{ Capítulos de libro } & Pre-seleccionados & Seleccionados & Empíricos & Documental \\
\hline El arte como terapia en el tratamiento del TDAH & 1 & 1 & 1 & 0 \\
$\begin{array}{l}\text { Diálogos entre arte y terapia del "arte psicótico" } \\
\text { al desarrollo de la arteterapia y sus aplicaciones }\end{array}$ & 1 & 0 & 0 & 0 \\
Arteterapia y Educación & 1 & 0 & 0 & 0 \\
Arteterapia en el Ámbito de la Salud Mental & 6 & 6 & 2 & 4 \\
\hline Total & 9 & 7 & 3 & 4 \\
\hline
\end{tabular}

Tabla 4

País de publicación

\begin{tabular}{|c|c|c|c|c|}
\hline País & Pre-seleccionados & Seleccionados & Empíricos & Documental \\
\hline Colombia & 6 & 3 & 1 & 2 \\
\hline Ecuador & 1 & 0 & 0 & 0 \\
\hline Chile & 6 & 3 & 3 & 0 \\
\hline Argentina & 2 & 1 & 0 & 1 \\
\hline Perú & 1 & 1 & 1 & 0 \\
\hline Costa Rica & 1 & 0 & 0 & 0 \\
\hline México & 2 & 0 & 0 & 0 \\
\hline Estados Unidos & 1 & 1 & 1 & 0 \\
\hline España & 52 & 34 & 23 & 11 \\
\hline Inglaterra & 1 & 1 & 0 & 1 \\
\hline \multirow[t]{2}{*}{ Total } & 73 & 44 & 29 & 15 \\
\hline & & & Total & 44 \\
\hline
\end{tabular}

Tabla 5

Problemáticas trabajadas

\begin{tabular}{lc}
\hline \multicolumn{1}{c}{ Problemática } & Frecuencia \\
\hline Trastorno del neurodesarrollo & 11 \\
\hline $\begin{array}{l}\text { Espectro de la esquizofrenia y } \\
\text { otros trastornos psicóticos }\end{array}$ & 8 \\
\hline $\begin{array}{l}\text { Trastornos depresivos } \\
\text { Mujeres inmigrantes en Estados Unidos }\end{array}$ & 6 \\
\hline Proceso de duelo & 1 \\
\hline $\begin{array}{l}\text { Expresiones simbólicas en comunidades } \\
\text { indígenas y afrocolombianas }\end{array}$ & 1 \\
\hline
\end{tabular}

Informes Psicológicos

Sele
. 
especializadas en la temática en Sur América. En general, la mayor parte de documentos seleccionados corresponden a investigaciones empíricas, lo cual permite evidenciar de forma más realista los beneficios obtenidos al trabajar con arteterapia.

La mayoría de documentos, tanto preseleccionados como seleccionados, no hacen una especificación exacta del enfoque utilizado para la intervención. Aun así, existen estudios que de forma no explícita evidencian principios de algún enfoque, resaltándose documentos con un enfoque más humanista. En otros casos, constructivista o con una orientación a la terapia Gestalt.

Con relación al tema, Marxen (2011) recuerda que existen diversas posturas psicoterapéuticas que han incorporado técnicas artísticas dentro de sus intervenciones. Un ejemplo son las junguianas, las cuales son de uso frecuente en Brasil y tienen como una de sus representantes más distinguidas a la británica Joy Schaverien. También está la perspectiva comportamental implementada por diferentes arteterapeutas estadounidenses.

Dentro de los documentos seleccionados se encontró un estudio de caso (Suarez, 2015) que evidencia la eficacia de las técnicas y algunos beneficios obtenidos. La intervención se realizó con un adolescente diagnosticado con Síndrome de Down que presentó un cuadro psicótico debido a una depresión no tratada. Allí, se realizó una intervención mediante sesiones divididas por fases. Las mejoras se observaron en la comunicación verbal, aumento de confianza y mayor expresividad emocional. Dichos resultados positivos se mantendrían tiempo después de terminar la terapia.

En relación con la psicomotricidad, los resultados arrojados en el estudio de Araujo y Gabelán (2010) indican que varios de los benéficos obtenidos se encuentran asociados a un incremento de la creatividad, comunicación y expresividad de la subjetividad, lo que posibilita el trabajar con factores conscientes e inconscientes. Sin embargo, mencionan que, para lograr estos resultados, se debe tener en cuenta que el abordaje teórico de la psicomotricidad sea compatible con el del arteterapia. Ya sea que la intervención se dé por sesiones grupales o individuales, los beneficios encontrados son similares. También es importante tener en cuenta técnicas generales como la escucha abierta, exploración de conflictos e incremento de la confianza (Orguillés, 2011). En conclusión, es relevante que se presenten beneficios para el consultante o paciente, pues sin beneficios no se podría lograr plasmar la eficacia.

Muchos de los documentos revisados tienen en común el trabajar especialmente con dibujo y pintura. Sin embargo, es importante tener en cuenta la problemática a trabajar con diferentes personas para saber que técnicas particulares se pueden incluir. Un ejemplo es el trabajo con adolescentes conflictivos y agresivos. Allí aparece el collage como una de las técnicas que proporciona más control para trabajar con este tipo de población. Lo anterior porque se trata de un proceso lento y controlable que consta de diferentes pasos (Marxen, 2011). 


\section{Consideraciones finales}

El arteterapia, más que ser una terapia propia de la psicología, ha buscado ser una terapia de ámbito interdisciplinar, por lo que muchos profesionales especializados en arteterapia provienen de carreras como Bellas Artes, Terapia Ocupacional, Trabajo Social, Psiquiatría y Enfermería. Lo anterior permite la emergencia de diversos puntos de vista para la intervención.

Los estudios encontrados proyectan la intervención en conjunto con un psicólogo, puesto que las problemáticas tratadas involucran trastornos mentales. De igual forma, no solo se relacionan con el campo de la salud mental, sino otras como la discapacidad, cuidados paliativos, oncología, pediatría y geriatría entre otros.

En cuanto a las limitaciones del estudio, no se pudieron revisar más documentos en español debido a la escasa producción de material en este idioma. Igualmente, aparecen algunas barreras económicas para la realización del estudio. Sin embargo, se lograron interesantes comprensiones relacionadas con este método de intervención. Con lo anterior se espera generar reflexiones que susciten preguntas de investigación para la realización de futuros estudios.

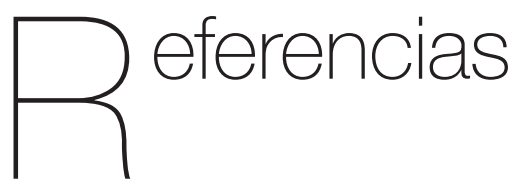

Araujo, G. \& Gabelán, G.N. (2010). Psicomotricidad y arteterapia. Revista Electrónica Interuniversitaria de Formación del Profesorado, 13(4), 307-319.
Aurora, R. \& Lara, M. (2000). La creatividad y la psicopatología. Revista Salud Mental, 25(5), 1-9.

Benito, E. (2009). Las psicoterapias. Psiencia, 1(1), 1-9. https://doi: 10.5872/ psiencia/10.2.21

Coll, F. (2015). Aplicación del arteterapia en el desarrollo de capacidades asociativas y cognitivas en personas con discapacidad psíquica (Tesis doctoral). Universidad de Murcia, Murcia.

Corbalán, J. (2006). La creatividad y su medida: matices y claves en arteterapia. En F. Coll. (Ed.), Arteterapia: dinámicas entre creación y procesos terapéuticos (pp. 45-56). Murcia, España: Universidad de Murcia.

Dumas, M. \& Aranguren, M. (2013). Beneficios del arteterapia sobre la salud mental. $V$ Congreso Internacional de Investigación y Práctica Profesional en Psicología XX Jornadas de Investigación Noveno Encuentro de Investigadores en Psicología del MERCOSUR. Facultad de Psicología - Universidad de Buenos Aires, Buenos Aires.

Fabres, G. (2011). Arteterapia y Esquizofrenia. Santiago de Chile, Chile: Universidad Santiago de Chile.

García, E. (2007). Tratamiento psicoterapéutico de los Trastornos Límite de Personalidad. Clínica y Salud, 18(3), 347-361. https://doi. org/10.1016/j.clysa.2017.09.003

García, J. (2018). Arteterapia: terapiapsicológica a través del arte. Recuperado el 27 de abril de 2018 de https://psicologiaymente.com/ clinica/arteterapia 
Hill (1945). Art Versus Illness. A Story Of Art Therapy. London: Allen and Unwin.

Hogan, S. (2001). Healing Arts: The History of Art Therapy. Londres, Reino Unido: Jessica Kingsley Publishers.

Junge, M. \& Asawa, P. (1994). A History of Art Therapy in the United States. Mundelein, Estados Unidos: American Art Therapy Association.

López, M. (2009). La Intervención Arteterapeutica y su Metodología en el Contexto Profesional Español (Tesis doctoral). Universidad de Murcia, Murcia.

Marxen, E. (2011). Diálogos entre arte y terapia: del "arte psicótico" al desarrollo de la terapia y sus aplicaciones. Barcelona: Gedisa.

Obradors, M. (2007). Creatividad y generación de ideas. Barcelona, España: Universitat Autònoma de Barcelona.

Orguillés, P. (2011). Arteterapia en las drogodependencias. Papeles de Arteterapia y Educación Artística para la Inclusión Social, 6, 135-149.

Sánchez, G. (2003). Creación, Arte y Psiquis. Bogotá, Colombia: Academia Nacional de Medicina de Colombia.

Strupp, H. H. (1978). The therapist's theoretical orientation: An overrated variable. Psychotherapy: Theory, Research \& Practice, 15(4), 314-317. http://dx.doi. org/10.1037/h0086020
Suarez, M. (2015). Arteterapia con un adolescente con síndrome de Down y psicosis. Contención a través de la experiencia artística (Trabajo de Especialización). Universidad de Chile, Santiago de Chile.

Vanegas, G., Barbosa, A. \& Pedraza, G. (2017). Revisión bibliográfia sobre el tratamiento sistémico y cognitivo conductual del trastorno límite de personalidad. Informes Psicológicos, 17(2), 159-176. http://dx.doi. org/10.18566/infpsic.v17n2a09

Vásconez, D. (2017). Aplicación de la técnica del Arteterapia como medio de intervención Terapéutica para disminuir el Trastorno de Ansiedad Generalizada en pacientes adultos mayores de edad comprendidas entre los 65 a 75 años que acuden al servicio de Terapia Ocupacional del Centro de Salud Tipo "C" de Guamaní en el periodo Abril-Septiembre 2017 (Trabajo de grado). Universidad Central del Ecuador, Quito.

Vecina, M. (2006). Creatividad. Revista Papeles del Psicólogo, 27(1), 31-29. https://doi. org/10.23923/pap.psicol2019.2877 